\title{
SELF-DUAL REPRESENTATIONS OF DIVISION ALGEBRAS AND WEIL GROUPS: A CONTRAST
}

\author{
DIPENDRA PRASAD AND DINAKAR RAMAKRISHNAN
}

\section{Introduction}

If $\rho$ is a selfdual representation of a group $G$ on a vector space $V$ over $\mathbb{C}$, we will say that $\rho$ is orthogonal, resp. symplectic, if $G$ leaves a nondegenerate symmetric, resp. alternating, bilinear form $B: V \times V \rightarrow$ $\mathbb{C}$ invariant. If $\rho$ is irreducible, exactly one of these possibilities will occur, and we may define a sign $c(\rho) \in\{ \pm 1\}$, taken to be +1 , resp. -1 , in the orthogonal, resp. symplectic, case.

Now let $k$ be a local field of characteristic 0 . The groups of interest to us will be $G=\mathrm{GL}_{m}(D)$, where $D$ is a division algebra with center $k$ and index $d$, and the Weil group $W_{k}$.

The local Langlands correspondence ([HT], [Hen1]) when used in conjunction with the Jacquet-Langlands correspondence ([Bdl]), gives a bijection $\pi \rightarrow \sigma$, satisfying certain natural properties, in particular the preservation of $\varepsilon$-factors of pairs, between the discrete series representations of $G$ and the set of irreducible representations $\sigma$ of $W_{k}^{\prime}$ of dimension $n=m d$. Here $W_{k}^{\prime}$ denotes $W_{k}$ if $k$ is Archimedean, and the extended Weil group $W_{k} \times \mathrm{SL}_{2}(\mathbb{C})$ if $k$ is non-Archimedean. One calls $\sigma$ the Langlands parameter of $\pi$. It is immediate from the construction that $\pi$ is selfdual if and only if $\sigma$ is. However, the local Langlands reciprocity is not a priori sensitive to the finer question of whether $c(\pi)$ equals $c(\sigma)$ or $-c(\sigma)$. The main result of this paper is the following.

Theorem A Let $n=m d, D$ a division algebra of index d over a local field $k$ of characteristic zero, $G=\mathrm{GL}_{m}(D)$, and $\pi$ an irreducible selfdual discrete series representation of $G$ with parameter $\sigma$ an irreducible representation of $W_{k}^{\prime}$ of dimension $n$. Then we have

$$
(-1)^{m} c(\pi)=(-1)^{n} c(\sigma)^{m} \text {. }
$$

Corollary B Let $\pi$ be an irreducible, selfdual representation of $D^{\times}$, for any division algebra $D$ of index $n$ over a local field $k$ of characteristic zero, and let $\sigma$ be the Langlands parameter of $\pi$. If $n$ is odd, $\pi$ is always orthogonal, while if $n$ is even,

$$
\pi \text { orthogonal } \Longleftrightarrow \sigma \text { symplectic. }
$$


When $n$ is odd, $\sigma$ is necessarily orthogonal, and so Theorem A implies, for any factorization $n=m d$ and $G=\mathrm{GL}_{m}(D)$ with $D$ a division algebra of index $d$, that $c(\pi)=+1$, i.e., $\pi$ is orthogonal. For $m=1$, we get $c(\pi)=(-1)^{n+1} c(\sigma)$, which implies that an irreducible selfdual representation $\pi$ of $D^{\times}, D$ of even index $n$, is symplectic if and only if $\sigma$ is orthogonal. This surprising flip is what we noticed first for $n=2$, spurring our interest in the general case, which is more subtle to establish. Based on considerations of Poincaré duality on the middle dimensional cohomology of certain coverings of the Drinfeld upper-half space, we conjectured in $[\mathrm{PR}]$ the assertion of Corollary $\mathrm{B}$, and established some positive results in $[\mathrm{PR}]$ and [Pra], including the case of $n=2$. In [Pra] it was proved that if $n$ is odd and if the residual characteristic of $k$ is odd, then $D^{\times}$has no selfdual irreducible representations of dimension $>1$, showing that in this case, the conjecture is difficult only for the even residual characteristic. A program to prove Corollary B along the geometric lines, using cohomological methods involving the formal moduli of Lubin-Tate groups, has been announced in the supercuspidal case by Laurent Fargues; it does not seem, however, that, without further input, his suggested methods would work for general discrete series representations, nor for $\mathrm{GL}_{m}(D)$.

Corollary B associates, to each irreducible, symplectic Galois representation $\sigma$ of dimension $n$ (even), a new secondary invariant, defined by whether, or not, the associated orthogonal representation $\pi$ of $D^{\times}$ lifts to the (s)pin group. This aspect was investigated for $n=2$ in $[\mathrm{PR}]$.

Our proof of Theorem A for non-Archimedean $k$ proceeds by using global methods, made possible by the following product formula.

Theorem C Let $F$ be a global field, $G=\mathrm{GL}_{m}(\mathbb{D})$, where $\mathbb{D}$ is a division algebra over $F$ and $Z$ the center of $G$. Suppose $\Pi=\otimes_{v}^{\prime} \Pi_{v}$ is an irreducible, selfdual automorphic representation of $G\left(\mathbb{A}_{F}\right)$ of central character $\omega$, which occurs with multiplicity one in the discrete part of $L^{2}\left(G(F) Z\left(\mathbb{A}_{F}\right) \backslash G\left(\mathbb{A}_{F}\right), \omega\right)$. Then we have

$$
\prod_{v \in \operatorname{ram}(\mathbb{D})} c\left(\Pi_{v}\right)=1,
$$

where $\operatorname{ram}(\mathbb{D})$ denotes the set of places where $\mathbb{D}$ is ramified.

As a consequence, we see that in the case $m=1$ and $\operatorname{ram}(\mathbb{D})=$ $\{u, v\}$, we have $c\left(\Pi_{u}\right)=c\left(\Pi_{v}\right)$, i.e., $\Pi_{u}$ and $\Pi_{v}$ are both orthogonal or both symplectic. In particular, if we know one, we know the other. Thanks to this product formula, given an irreducible selfdual representation $\pi$ of $D^{\times}$, with $D$ a division algebra over a local field $k$, our 
strategy becomes one of finding a number field $F$ with $F_{v}=k$ for a place $v$ of $F$, a division algebra $\mathbb{D}$ over $F$ with $\operatorname{ram}(\mathbb{D})=\{u, v\}$, and a selfdual automorphic representation $\Pi$ of $\mathbb{D}^{\times}\left(\mathbb{A}_{F}\right)$ such that $\Pi_{v}=\pi$ and $\Pi_{u}$ a representation for which Theorem A can be checked. Thanks to the Jacquet-Langlands correspondence between $\mathbb{D}^{\times}$and $\mathrm{GL}_{n}$, we see that it suffices to find a selfdual discrete automorphic representation $\Pi^{\prime}$ of $\mathrm{GL}_{n}\left(\mathbb{A}_{F}\right)$ with $\Pi_{v}^{\prime}$, resp. $\Pi_{u}^{\prime}$, being associated to $\Pi_{v}$, resp. $\Pi_{u}$. The difficulty is not so much in globalizing, but in choosing a global $\Pi^{\prime}$ which is also selfdual. We have been able to address this, when $\Pi_{u}$ and $\Pi_{v}$ are both supercuspidal by using various known instances of Functoriality, culminating in the works of Jiang and Soudry ([JS2], [Sou1]). In section 3 we check Theorem A for representations of $D^{\times}$ trivial on $D^{\times}(1)$, which can be used at the place $u$, allowing foothold on all representations.

This strategy works with only minor modifications for $\mathrm{GL}_{m}(D)$ too; when the parameter of the representation on the Galois side is nontrivial on $\mathrm{SL}_{2}(\mathbb{C})$, we use Moeglin-Waldspurger's description ([MW]) of residual spectrum, on which the general Jacquet-Langlands correspondence has been established by Badulescu [Bdl]).

The statement of Theorem A for $n$ odd is the simplest as it does not refer to the Langlands parameter at all: $c(\pi)=1$ for any irreducible selfdual discrete series representation of $\mathrm{GL}_{m}(D)$ with $n=m d$ odd. It says in particular that a selfdual irreducible representation of $D^{\times}$ for a division algebra of index $n$, an odd integer, must be orthogonal. Since $D^{\times} / k^{\times}$is a profinite group, the question is clearly in the realm of finite group theory. However, our proof uses many recent and nontrivial results in the theory of Automorphic representations to achieve this. The recent preprint of Bushnell and Henniart has given a local proof of this result for $D^{\times}$in $[\mathrm{BH}]$.

The first author would like to thank the California Institute of Technology for the invitation to visit, enabling the authors to collaborate on this work. He thanks the Institute for Advanced Study where a significant portion of this work was done, and gratefully acknowledges receiving support through grants to the Institute by the Friends of the Institute, and the von Neumann Fund, and also thanks the Clay Math Institute for supporting him at the final stages of this work. He would also like to thank E. Lapid for his interest and for suggesting a similar approach for $G=D^{\times}$. Both the authors would like to thank J. Bernstein, for inquiring about $\mathrm{GL}_{m}(D)$, as well as I. Badulescu, L. Clozel, M. Harris, G. Henniart, D. Soudry, and W. Zink for making helpful comments. The second author would in addition like to thank Hervé 
Jacquet and Akshay Venkatesh for their interest, and also acknowledge support from the National Science Foundation through the grant DMS-0701089.

\section{Results on functoriality which we need}

We have the following theorem due to Jiang and Soudry, cf. Theorem 6.4 of [JS1], and Theorem 2.1 of [JS2]. We are thankful to David Soudry for writing one of us ([Sou2]) explaining the completion of their work; see also [Sou1] and [GJR].

Theorem 1.1. Let $G$ be either a symplectic group or a quasi-split orthogonal group over a non-Archimedean local field $k$. Let the $L$ group of $G$ come equipped with its natural representation into $\mathrm{GL}_{n}(\mathbb{C})$. Then there exists a natural bijective correspondence between irreducible generic discrete series representations of $G(k)$ and irreducible generic representations of $\mathrm{GL}_{n}(k)$, with Langlands parameter of the form

$$
\sigma=\sum \sigma_{i}
$$

where $\sigma_{i}$ are pairwise inequivalent, irreducible representations of $W_{k}^{\prime}$ which are all orthogonal except when $G$ is an odd orthogonal group in which case they are all symplectic; if $G$ is an even orthogonal group, quasi-split over $k$, and split over a quadratic extension $K$ of $k$, then we assume that $\operatorname{det} \sigma=\omega_{K / k}$, where $\omega_{K / k}$ is the quadratic character of $k^{\times}$ associated to $K$.

The following theorem of Cogdell, Kim, Piatetski-Shapiro, and Shahidi (cf. [CPSS], [CKPSS]) establishes the weak Langlands functoriality from the generic cusp forms on $G$ to $\mathrm{GL}_{n}$; the last part of the theorem, strengthening weak Langlands functoriality to be Langlands functorial at all places is due to Jiang and Soudry, cf. Theorem E of [JS2] when $G=\mathrm{SO}(2 m+1)$, and [Sou1], [Sou2] for the remaining cases; a general statement is also in [GJR].

Theorem 1.2. Let $G$ be either a symplectic group or a quasi-split orthogonal group over a number field $K$. Let $\mathcal{A}^{0, g}\left(G\left(\mathbb{A}_{K}\right)\right)$ be the set of irreducible generic cuspidal automorphic representations of $G\left(\mathbb{A}_{K}\right)$, and let $\mathcal{A}\left(\mathrm{GL}_{n}\left(\mathbb{A}_{K}\right)\right)$ be the set of irreducible automorphic representations of $\mathrm{GL}_{n}\left(\mathbb{A}_{K}\right)$. Then there is a weak functorial transfer from $\mathcal{A}^{0, g}\left(G\left(\mathbb{A}_{K}\right)\right)$ to $\mathcal{A}\left(\mathrm{GL}_{n}\left(\mathbb{A}_{K}\right)\right)$; it is in fact Langlands functorial at every place of $K$.

An implication for us of these theorems is the following:

Theorem 1.3. Let $K$ be a number field, and $v_{i}, i=1, \cdots, d$ places of $K$. Let $K_{v_{i}}$ be the corresponding local fields. Suppose that the $\pi_{i}$ are 
irreducible selfdual supercuspidal representations of $\mathrm{GL}_{n}\left(K_{v_{i}}\right)$ whose parameters are either orthogonal, or symplectic for all $i$. Then there exists a selfdual cuspidal automorphic representation $\pi$ on $\mathrm{GL}_{n}\left(\mathbb{A}_{K}\right)$ with $\pi_{i}$ as the local component of $\pi$ at each of the places $v_{i}$.

Proof: Since the parameters of the representations $\pi_{i}$ are all orthogonal or are all symplectic, they can be transported, thanks to Theorem 1.1, to generic supercuspidal representations $\Pi_{i}$ of $G\left(K_{v_{i}}\right)$ for a quasisplit orthogonal or a symplectic group $G$ over a number field $K$. These can be globalized into a cuspidal generic automorphic representation $\Pi$ on $G\left(\mathbb{A}_{K}\right)$, i.e., with $\Pi_{v_{i}} \simeq \Pi_{i}$ for all $i$ by a theorem due to Vignéras [Vig]. The automorphic representation $\Pi$ can then be transferred to $\mathrm{GL}_{n}$ by Theorem 1.2, such that the lifted automorphic representation $\pi$ on $\mathrm{GL}_{n}\left(\mathbb{A}_{K}\right)$ has the correct local components $\pi_{i}$ at all the $v_{i}$ by Theorem 1.2, proving the theorem.

Finally, the following form of the Jacquet-Langlands correspondence between $\mathrm{GL}(n)$ and its inner forms, due to Badulescu, cf. [Bad], will also play crucial role for us in the proof of Theorem A.

Theorem 1.4. (Badulescu-Jacquet-Langlands) Let $\Pi$ be an automorphic representation of $\mathrm{GL}_{n}\left(\mathbb{A}_{K}\right)$ appearing in the discrete spectrum. Suppose $\mathbb{B}$ is a central simple algebra over $K$ of dimension $n^{2}$. Let $S$ be the finite set of places where $\mathbb{B}$ is not split. Assume $S$ has only non-Archimedean places. Then $\Pi$ can be transferred to an automorphic representation $\Pi^{\prime}$ on $\mathbb{B}^{\times}\left(\mathbb{A}_{K}\right)$ if at every place $v$ in $S, \Pi_{v}$ is either a discrete series representation or is a generalized Speh representation. The representations $\Pi_{v}$ and $\Pi_{v}^{\prime}$ either have the same parameters, or are related to each other by the Aubert-Zelevinsky involution. Any automorphic representation of $\mathbb{B}^{\times}\left(\mathbb{A}_{K}\right)$ appears with multiplicity 1 in the discrete spectrum.

\section{Orthogonality and Reality}

The following basic Lemma is presumably well known, but for lack of an appropriate reference outside the realm of compact groups, we supply a proof.

Lemma 2.1. An irreducible admissible, unitary representation $(\pi, V)$ of a p-adic group $G$ carries a nonzero symmetric bilinear form $B$ : $V \times V \rightarrow \mathbb{C}$ if and only if $\pi$ is defined over $\mathbb{R}$, i.e., there is a $G$ invariant real subspace $W$ of $V$ such that $V=W \otimes_{\mathbb{R}} \mathbb{C}$. 
Proof : Recall that a complex vector space $V$ is defined over $\mathbb{R}$ if and only if there is a complex conjugation on $V$, i.e., an involution $v \rightarrow \bar{v}$ on $V$ such that $\overline{z v}=\bar{z} \bar{v}$.

Let $H: V \times V \rightarrow \mathbb{C}$ be a positive definite Hermitian form on $V$. We will prove that if $V$ is defined over $\mathbb{R}$ (and therefore has a complex conjugation: $v \rightarrow \bar{v}$ ), then $V$ carries a nonzero $G$-invariant symmetric bilinear form. Define,

$$
B(v, w)=H(v, \bar{w}) .
$$

Clearly, $B(\lambda v, \mu w)=\lambda \mu B(v, w)$ for $\lambda, \mu \in \mathbb{C}$; further $B$ is a $G$-invariant nonzero bilinear form. It remains to check that $B$ is symmetric, which will follow if we can prove that

$$
H(v, w)=\overline{H(\bar{v}, \bar{w})}
$$

for $v, w \in V$.

In any case, by the uniqueness (up to a positive real number) of a positive definite Hermitian form on $V$, there exists a $\lambda>0$ such that

$$
H(v, w)=\lambda \overline{H(\bar{v}, \bar{w})} .
$$

Since $\overline{\bar{v}}=v, \lambda^{2}=1$, which means that $\lambda=1$ as it is positive, proving that $B$ is symmetric.

Conversely, we show that if there exists a symmetric $G$-invariant bilinear form $B: V \times V \rightarrow \mathbb{C}$, then $V$ is defined over $\mathbb{R}$, by constructing an involution $v \rightarrow \bar{v}$ on $V$ (which is $G$-invariant, and conjugate linear).

This part of the proof will use the fact that any smooth linear form on $V$, meaning a linear form which is left invariant by a compact open subgroup of $G$, is of the form $v \rightarrow H(v, w)$ for a unique $w$ in $V$. This allows us to define a map, $w \rightarrow w^{\prime}$ on $V$ by

$$
B(v, w)=H\left(v, w^{\prime}\right),
$$

for all $v, w \in V$. Clearly, $w \rightarrow w^{\prime}$ is conjugate linear. Using the fact that $B$ is symmetric, it is easy to see that $w \rightarrow w^{\prime}$ is an involution if and only if

$$
H(v, w)=\overline{H\left(v^{\prime}, w^{\prime}\right)} .
$$

However, this is not true in general. In any case, by uniqueness (up to a positive real number) of positive definite Hermitian forms,

$$
H(v, w)=\lambda \overline{H\left(v^{\prime}, w^{\prime}\right)}
$$

for some $\lambda>0$. It is then easy to check that $v \rightarrow \bar{v}=\sqrt{\lambda} v^{\prime}$ is an involution, giving rise to a real structure on $V$, proving the lemma. 
Corollary 2.2. Let $(\pi, V)$ be an irreducible unitary representation of a p-adic group $G$, and $H$ a closed subgroup of $G$. Assume that the space of linear forms $\ell: V \rightarrow \mathbb{C}$ which are $H$-invariant is one dimensional. Then if $\pi$ is selfdual, it is orthogonal.

Proof : It suffices to prove that the representation $(\pi, V)$ of $G$ can be defined over $\mathbb{R}$. This is equivalent to proving that there is a conjugate linear automorphism $\varphi$ of $V$ commuting with the $G$-action with $\varphi^{2}=1$. Since $V$ is unitary and selfdual, $V$ is isomorphic to $\bar{V}$, and therefore there is a conjugate linear automorphism $\phi$ of $V$ which by Schur's lemma has $\phi^{2}=\mu$ for some $\mu$ in $\mathbb{C}^{\times}$. We will prove that $\phi$ can be scaled to achieve $\mu=1$.

Because of the uniqueness of the $H$-invariant linear form $\ell$,

$$
\overline{\ell(v)}=\lambda \ell(\phi(v)),
$$

for all $v \in V$ (for some $\lambda \in \mathbb{C}^{\times}$). Applying this identity to $\phi(v)$ instead of $v$, and noting that $\phi^{2}(v)=\mu \cdot v$, we find that

$$
\bar{\lambda}^{-1} \ell(v)=\lambda \mu \ell(v) .
$$

Therefore $\mu^{-1}=\lambda \bar{\lambda}$, and changing $\phi$ to $\varphi=\lambda \phi$, we have $\varphi^{2}=1$, proving the corollary.

Corollary 2.3. Let $\pi_{1}$ be an irreducible unitary selfdual representation of a p-adic group $G, H$ a closed subgroup of $G$, and $\pi_{2}$ an irreducible unitary selfdual representation of $H$ such that $\operatorname{Hom}_{H}\left(\pi_{1}, \pi_{2}\right) \cong \mathbb{C}$. Then the parities of $\pi_{1}$ and $\pi_{2}$ are the same.

Proof : The previous corollary applied to the representation $\pi_{1} \otimes$ $\pi_{2}^{\vee}$ of the group $G \times H$ containing the subgroup $H \times H$ proves this assertion.

\section{The Product Formula: Proof of Theorem C}

Preserving the notations of Theorem $\mathrm{C}$, define a $G\left(\mathbb{A}_{F}\right)$-invariant bilinear form $B$ on $\Pi$ by

$$
(f, g) \longrightarrow \int_{G(F) Z\left(\mathbb{A}_{F}\right) \backslash G\left(\mathbb{A}_{F}\right)} f g d \mu, \quad \forall f, g \in \Pi,
$$

where $d \mu$ is an invariant measure on $G(F) Z\left(\mathbb{A}_{F}\right) \backslash G\left(\mathbb{A}_{F}\right)$. We check that this is a non-degenerate bilinear form on $\Pi$. Note that the space of functions spanned by $\bar{f}$ (the complex conjugate of $f$ ), as $f$ varies over $\Pi$, gives rise to the representation $\Pi^{\vee}$, which is isomorphic to $\Pi$. Hence by the multiplicity $1, \bar{f} \in \Pi$. Since

$$
B(f, \bar{f})=\int f \bar{f} d \mu \neq 0,
$$


the bilinear form $B$ on $\Pi$ is non-degenerate. It is evidently symmetric too, proving that $c(\Pi)=1$.

Now $c\left(\Pi_{v}\right)=1$ for all places $v$ where the division algebra $D$ is unramified is a consequence of the following result, proving Theorem C.

Proposition 3.1. Every irreducible admissible selfdual representation $\pi$ of $\mathrm{GL}_{n}(k)$ over any local field $k$ is orthogonal.

Proof: First let $k$ be non-Archimedean. Let $U(k)$ be the subgroup of $\mathrm{GL}_{n}(k)$ consisting of upper-triangular unipotent matrices. By a theorem of Zelevinsky ([Zlv], Corollary to Theorem 8.1), for any representation $\pi$ of $\mathrm{GL}_{n}(k)$, there is a character $\psi: U(k) \rightarrow \mathbb{C}^{\times}$which appears in $\pi$ as a quotient with multiplicity 1 . We may assume that $\pi$ is non-trivial, which forces $\psi$ to be non-trivial. Since $\psi$ is not selfdual, we cannot directly apply Corollary 2.3 , but a small modification works. Let $s$ be the diagonal element $\left(1,-1,1, \cdots,(-1)^{n-1}\right)$. The 2-dimensional representation $\tau$ of $H:=\langle s\rangle \cdot U(k)$ induced from the character $\psi$ of $U(k)$ is an irreducible orthogonal representation. So Corollary 2.3 applies, with $\pi_{1}=\pi$ and $\pi_{2}=\tau$, to yield the desired conclusion.

It is left to prove the Proposition when $k$ is Archimedean.

It is a general result due to D.Vogan [Vo] that any irreducible $(\mathfrak{g}, K)$ module has a minimal $K$-type which occurs with multiplicity 1 . For $\mathrm{GL}_{n}(\mathbb{R})$ and $\mathrm{GL}_{n}(\mathbb{C})$, the minimal $K$-type is unique -the authors owe this remark to D. Vogan- and therefore a selfdual representation of these groups has a selfdual minimal $K$-type. From the following wellknown lemma, every irreducible, selfdual representation of the maximal compact subgroup $\mathrm{O}_{n}(\mathbb{R})$ (resp. $\mathrm{U}_{n}(\mathbb{R})$ ) carries an invariant symmetric bilinear form, which concludes the proof of this lemma. (Note the difference between $\mathrm{U}_{n}(\mathbb{R})$ and $\mathrm{SU}_{n}(\mathbb{R})$; there are already irreducible symplectic representations of $\mathrm{SU}_{2}(\mathbb{R})$, but they do not extend to selfdual representations of $\mathrm{U}_{2}(\mathbb{R})$.)

Lemma 3.2. Every irreducible, selfdual representation of $\mathrm{U}_{n}(\mathbb{R})$ is orthogonal. Every irreducible representation of $\mathrm{O}_{n}(\mathbb{R})$ is selfdual and orthogonal.

Proof : We supply one of the many possible proofs for the convenience of the reader. For a compact connected Lie group $G$ with maximal torus $T$, and $w_{0}$ the longest element in the Weyl group of $T$ (with respect to some ordering of positive roots), the dual of a finite dimensional irreducible representation $\pi_{\lambda}$ with highest weight $\lambda$ is $\pi_{-w_{0}(\lambda)}$. For $\mathrm{U}_{n}(\mathbb{R})$, let $T$ be the diagonal torus, and $w_{0}\left(t_{1}, t_{2}, \cdots, t_{n}\right)=\left(t_{n}, \cdots, t_{2}, t_{1}\right)$. 
Therefore highest weights of irreducible selfdual representations are of the form:

$$
\lambda_{1} \geq \lambda_{2} \geq \cdots \geq-\lambda_{2} \geq-\lambda_{1}
$$

By the well-known branching law from $\mathrm{U}_{n}(\mathbb{R})$ to $\mathrm{U}_{n-1}(\mathbb{R})$, cf. [Zlb], section 132, pp. 385-387, we find that an irreducible selfdual representation of $\mathrm{U}_{n}(\mathbb{R})$ contains an irreducible selfdual representation of $\mathrm{U}_{n-1}(\mathbb{R})$ with multiplicity one; therefore by induction on $n$, every irreducible selfdual representation of $\mathrm{U}_{n}(\mathbb{R})$ is orthogonal.

For $\mathrm{SO}_{2 n+1}(\mathbb{R}), w_{0}=-1$, so every irreducible representation is selfdual. For $\mathrm{SO}_{2 n}(\mathbb{R})$, the inner conjugation action of $\mathrm{O}_{2 n}(\mathbb{R})$ takes a representation of $\mathrm{SO}_{2 n}(\mathbb{R})$ to its dual, hence every irreducible representation of $\mathrm{O}_{2 n}(\mathbb{R})$ is selfdual. By the (multiplicity freeness of) branching from $\mathrm{O}_{n}(\mathbb{R})$ to $\mathrm{O}_{n-1}(\mathbb{R})$, cf. [Zlb], section 129, Corollaries of Thms. 2, 3, pp. 378-9, we are done again.

This completes the proof of Theorem C.

\section{Sign in the Level 1 case}

In this section we prove Theorem A about irreducible selfdual representations of $D^{\times} / D^{\times}(1)$. The global proofs in this paper involve a reduction step using the product formula, and this approach depends crucially, in most situations, on this local input, which represents the simplest of the situations for Theorem A.

Various aspects of the representation theory of $D^{\times} / D^{\times}(1)$ are analyzed in the work of Silberger and Zink in [SZ]. We begin with some notation, and recalling the parametrization of the irreducible representations of $D^{\times} / D^{\times}(1)$ from [SZ] (called level zero representations there).

As in the rest of the paper, let $D$ be a division algebra with center a non-Archimedean local field $k$, and of index $n$. Let $n=e f$, and let $k_{f}$ be the unramified extension of $k$ of degree $f$, contained in $D$. Let $D_{f}$ be the centralizer of $k_{f}$ in $D$ which is a division algebra with center $k_{f}$ and of index $e$. A character $\chi$ of $k_{f}^{\times}$will be called regular if all its Galois conjugates are distinct. For a character $\chi$ of $k_{f}^{\times}$, let $\tilde{\chi}$ be the character of $D_{f}^{\times}$obtained by composing $\chi$ with the reduced norm mapping Nrd : $D_{f}^{\times} \rightarrow k_{f}^{\times}$. If the character $\chi$ is tame, i.e., trivial on $k_{f}^{\times}(1)$, then the character $\tilde{\chi}$ of $D_{f}^{\times}$can be extended to a character of $D^{\times}(1) D_{f}^{\times}$by declaring it to be trivial on $D^{\times}(1)$, which, by abuse notation, we again denote by $\tilde{\chi}$.

With this notation, it follows from Clifford theory as in [SZ] that the dimensions of irreducible representations of $D^{\times} / D^{\times}(1)$ are divisors of $n$, and that there is a bijection between irreducible representations 
of $D^{\times} / D^{\times}(1)$ of dimension $f$ and regular characters of $k_{f}^{\times}$which are trivial on $k_{f}^{\times}(1)$ (modulo the action of the Galois group of $k_{f}$ over $k$ on the set of such characters) obtained by inducing the character $\tilde{\chi}$ of $D^{\times}(1) D_{f}^{\times}$to $D^{\times}$.

We analyze these representations to see if they are orthogonal or symplectic in the following general proposition.

Proposition 4.1. Let $N$ be a normal subgroup of a group $G$ of index $f>1$, such that $G / N$ is a cyclic group of order $f$. Let $\varpi$ be an element of $G$ whose image in $G / N$ is a generator of the cyclic group $G / N$. Let $\pi$ be an irreducible representation of $G$ of dimension $f$ whose restriction to $N$ contains a character $\chi: N \rightarrow \mathbb{C}^{\times}$, so that $\pi=\operatorname{Ind}_{N}^{G} \chi$. Then the following hold:

(1) If $\pi$ is selfdual, $f$ is even, say $f=2 d$.

(2) The representation $\pi$ is selfdual of dimension $f=2 d$ if and only if $\chi^{-1}=\chi^{<d>}$, where $\chi^{<d>}(n)=\chi\left(\varpi^{d} n \varpi^{-d}\right)$ for $n \in N$.

(3) If $\pi$ is selfdual, $\chi\left(\varpi^{f}\right)= \pm 1$, and $\chi\left(\varpi^{f}\right)=1$ if and only if $\pi$ is an orthogonal representation.

(4) If $\pi$ is selfdual, it is orthogonal if and only if $\operatorname{det} \pi(\varpi)=-1$.

Proof : Since $\pi$ is irreducible, all the conjugates $\chi^{<i>}$ of $\chi$, defined by $\chi^{<i>}(n)=\chi\left(\varpi^{i} n \varpi^{-i}\right)$ are distinct for $i=0,1, \cdots, f-1$. The representation $\pi=\operatorname{Ind}_{N}^{G} \chi$ is selfdual if and only if $\operatorname{Ind}_{N}^{G} \chi=\operatorname{Ind}_{N}^{G} \chi^{-1}$, i.e., if and only if $\chi^{-1}=\chi^{<i>}$ for some $i$. This implies that $2 i \equiv 0$ $\bmod f$, which means that $f$ must be even, say $f=2 d$, and $i=d$.

As the element $\varpi^{f}$ commutes with $\varpi, \chi^{<i>}\left(\varpi^{f}\right)=\chi\left(\varpi^{f}\right)$ for all $i$. Therefore $\varpi^{f}$ operates on $\pi$ by a scalar, which, if $\pi$ is selfdual, must be \pm 1 .

Let $e_{0}$ be any vector in the space of $\pi$ on which $N$ operates via the character $\chi$. Define $\varpi^{i} \cdot e_{0}=e_{i}$ for $0 \leq i \leq f-1$. If $\varpi^{f} \cdot e_{0}=-e_{0}$, then

$$
e_{0} \wedge e_{d}+e_{1} \wedge e_{d+1}+\cdots+e_{d-1} \wedge e_{2 d-1}
$$

is left invariant under both $\varpi$ and $N$, hence the representation is symplectic. If, however, $\varpi^{f} \cdot e_{0}=e_{0}$, then the vector

$$
e_{0} \cdot e_{d}+e_{1} \cdot e_{d+1}+\cdots+e_{d-1} \cdot e_{2 d-1}
$$

in $S^{2}(\pi)$ is left invariant under both $\varpi$ and $N$, hence the representation is orthogonal.

Since $\varpi e_{i}=e_{i+1}$ for $i \in \mathbb{Z} / f$, if and only if $\pi$ is orthogonal, it implies that if $\pi$ is selfdual, it is orthogonal if and only if $\operatorname{det} \pi(\varpi)=-1$.

Continuing now with the representations of $D^{\times}$of dimension $f$ with ef $=n$, let $\varpi$ be an element of $D^{\times}$which normalizes $k_{n}$, an unramified 
extension of $k$ inside $D$ of degree $n$ over $k$, such that $\varpi^{n}=\varpi_{k}$, a uniformizer in $k$. The element $\varpi$ of $D^{\times}$projects to a generator of the cyclic group $D^{\times} / D^{\times}(1) D_{f}^{\times} \cong \mathbb{Z} / f \mathbb{Z}$, and as $\varpi^{f}$ centralizes $k_{f}$, it lies in $D_{f}$. Since $\left(\varpi^{f}\right)^{e}=\varpi_{k}$, it follows that the reduced norm of $\varpi^{f}$ is $(-1)^{e-1} \varpi_{k}$. From the previous proposition, we conclude the following corollary.

Corollary 4.2. Let $\chi$ be a regular tame character of $k_{f}^{\times}$, and $\pi_{\chi}$ the associated representation of $D^{\times}$of dimension $f$. Then $\pi_{\chi}$ is selfdual if and only if $f$ is even, say $f=2 d$, and the character $\chi$ restricted to $k_{d}^{\times}$is trivial on the index 2 subgroup consisting of norms from $k_{f}^{\times}$. Assuming $\pi_{\chi}$ to be selfdual, it is orthogonal if and only if $\chi$ restricted to $k_{d}^{\times}$is trivial.

Recall that the Weil group $W_{k_{f} / k}$ sits in the exact sequence,

$$
1 \rightarrow k_{f}^{\times} \rightarrow W_{k_{f} / k} \rightarrow \operatorname{Gal}\left(k_{f} / k\right) \rightarrow 1
$$

As there is an element $\varpi$ in $W_{k_{f} / k}$ which goes to the generator of the Galois group of $k_{f}$ over $k$, and whose $f$-th power is a uniformizer in $k$, we have the following corollary for representations of the Weil group.

Corollary 4.3. For a regular character $\mu$ of $k_{f}^{\times}$, let $\sigma_{\mu}$ be the induced representation of $W_{k_{f} / k}$ of dimension $f$. Then $\sigma_{\mu}$ is selfdual if and only if $f$ is even, say $f=2 d$, and the character $\mu$ restricted to $k_{d}^{\times}$is trivial on the index 2 subgroup consisting of norms from $k_{f}^{\times}$. Assuming $\sigma_{\mu}$ to be selfdual, it is orthogonal if and only if $\mu$ restricted to $k_{d}^{\times}$is trivial, or if and only if $\operatorname{det} \sigma_{\mu}$ is nontrivial.

The following result, correcting a mistake in the Remark on page 182 of the paper of Silberger and Zink [SZ], was proposed by us to G. Henniart, and has since been proved by Bushnell and Henniart; see their forthcoming paper. This completes the proof of theorem A for representations of $D^{\times}$which are trivial on $D^{\times}(1)$. In this theorem, and in what follows we will let $s p_{e}$ denote the $e$-dimensional irreducible representation of $\mathrm{SL}_{2}(\mathbb{C})$.

Theorem 4.4. The Langlands parameter of the representation $\pi_{\chi}$ of dimension $f$ of $D^{\times}$is $\sigma_{\mu} \otimes s p_{e}$, with $\sigma_{\mu}$ the $f$-dimensional representation of $W_{k}$ induced by the character

$$
\mu:=\chi \omega_{2}^{e(f-1)}: k_{f}^{\times} \rightarrow \mathbb{C}^{\times},
$$

where $\omega_{2}$ is the quadratic unramified character of $k_{f}^{\times}$.

Remark 4.5: In order to be able to make use of representations of level 1, we must know that there are irreducible orthogonal as well as 
symplectic representations of $D^{\times} / D^{\times}(1)$ of dimension $f$ for any even divisor $f=2 d$ of $n$. By corollary 4.2, this reduces to a question over finite fields. A character of $\mathbb{F}_{q^{2 d}}^{\times}$extended to a tame character of $k_{f}^{\times}$ with values \pm 1 on the uniformizer gives rise to an irreducible selfdual representation of $D^{\times}$of dimension $f$ if and only if it arises from the group of norm 1 elements of $\mathbb{F}_{q^{2 d}}^{\times}$, denoted $\mathbb{S}^{1}\left(\mathbb{F}_{q^{d}}\right)$, through the map $x \rightarrow x / \bar{x}$ for $x \in \mathbb{F}_{q^{2 d}}^{\times}$, but does not factor through a smaller field under the norm mapping. Since $\mathbb{S}^{1}\left(\mathbb{F}_{q^{d}}\right)$ is a cyclic group of order $\left(q^{d}+1\right)$, one can consider characters on it of order $\left(q^{d}+1\right)$. Such characters of $\mathbb{F}_{q^{2 d}}^{\times}$ will not arise from an intermediate field through the norm mapping as those have orders divisible by $\left(q^{s}-1\right)$, where $s$ is a divisor of $2 d$.

\section{Proof of Theorem A}

We now prove Theorem A in the following equivalent form.

Theorem 5.1. Let $n \geq 1, n=m d, k$ a non-Archimedean local field, $D$ a division algebra over $k$ of index $d$, and $\pi^{\prime}$ an irreducible selfdual representation of $G L_{m}(D)$. Then $\pi^{\prime}$ is orthogonal if $d$ is odd. If $d$ is even, and $m$ is odd, then $\pi^{\prime}$ is orthogonal if and only if its parameter $\sigma$ is symplectic. If both $m$ and $d$ are even, then the representation $\pi^{\prime}$ is orthogonal.

Proof. Write the parameter $\sigma$ (of $\pi^{\prime}$ ) as $\tau \otimes s p_{b}$, with $a b=n$ and $\tau$ an irreducible, selfdual $a$-dimensional representation of $W_{k}$. The proof divides naturally into three cases, according to whether $b=0$, or $b>0$ with $a$ is even, or $b>0$ with $a$ odd. Denote by $\pi$ the irreducible selfdual representation of $\mathrm{GL}_{n}(k)$ corresponding to $\pi^{\prime}$ by the local Jacquet-Langlands correspondence.

Case I: $\quad \mathbf{b}=\mathbf{0}$ : In this case $\sigma$ is trivial on $\mathrm{SL}_{2}(\mathbb{C})$, and so $\pi$ is supercuspidal.

Suppose $d$ is odd. As $\pi$ is selfdual and supercuspidal, we may globalize it, by applying Theorem 1.3, to a selfdual, cuspidal automorphic representation $\Pi$ of $\mathrm{GL}_{n}\left(\mathbb{A}_{K}\right)$ with $\pi$ as its local component at $K_{v}=k$. Let $E$ be a cyclic extension of $K$ of degree $d$ such that $v$ splits into $d$ places. Let $\Pi_{E}$ denote the base change of $\Pi$ to $E$. Let $\mathbb{B}$ be a central division algebra over $E$ of dimension $d^{2}$ over $E$ such that $\mathbb{B} \otimes_{K} K_{v} \cong D^{d}$, and such that $\mathbb{B}$ has no other ramification. That there is such a division algebra $\mathbb{B}$ follows from classfield theory.

Let $\Pi_{E}^{\mathbb{B}}$ denote the automorphic representation of $\mathrm{GL}_{m}\left(\mathbb{B}\left(\mathbb{A}_{E}\right)\right)$ obtained from $\Pi_{E}$ by the Jacquet-Langlands correspondence ([Bdl]). The component of $\Pi_{E}^{D}$ at $E_{v}:=E \otimes_{K} K_{v} \simeq k^{d}$ is isomorphic to $\pi^{\prime \otimes d}$. At 
every place $u$ of $E$ not lying over $v$, the $u$-component of $\Pi_{E}^{\mathbb{B}}$ is a representation of $\mathrm{GL}_{n}\left(E_{u}\right)$. Applying Theorem 3.1, we find that the $d$-th power of $c\left(\pi^{\prime}\right)$ is trivial, and therefore $\pi^{\prime}$ is an orthogonal representation (as $d$ is odd).

We are left to consider when $d$ is even. Write the index as $d=2 r$, and the Brauer invariant of $D$ as $\frac{s}{2 r} \in \mathbb{Q} / \mathbb{Z}$ with $(s, 2 r)=1$. Let $\mathbb{D}$ be a division algebra over a number field $K$ of index $2 m r$ such that $\mathbb{D}$ gives rise to $\mathrm{M}_{m}(D)$ at one place $v$, and with Brauer invariants $\frac{-1}{2 m r} \in \mathbb{Q} / \mathbb{Z}$ at $s m$ other places, call them $u_{1}, \ldots, u_{s m}$. We may further take $\mathbb{D}$ to be split at all the remaining places. The existence of such a global division algebra follows from classfield theory.

Globalize $\pi$ to an automorphic representation $\Pi$ of $\mathrm{GL}_{n}\left(\mathbb{A}_{K}\right)$ such that its local components at the places $u_{1}, \ldots, u_{s m}$ correspond to representations of $\mathbb{D}_{u_{i}}^{\times}$trivial on $\mathbb{D}_{u_{i}}^{\times}(1)$, and selfdual of the same parity as $\pi$ (parity to be understood on the Galois side). Transporting this automorphic representation to $\mathbb{D}^{\times}\left(\mathbb{A}_{K}\right)$, and using the product formula given in Theorem 3.1, we get

$$
c(\pi)=[-c(\sigma)]^{s m}=[-c(\sigma)]^{m},
$$

the last equality due to the fact that $s$ is odd because of the condition earlier $(s, 2 r)=1$. This is equivalent to the conclusion of the theorem, therefore proving it when $\pi$ is supercuspidal.

\section{Case II: $\quad b \neq 0$ and a even:}

Let $\Sigma$ be a selfdual cuspidal automorphic representation of $\mathrm{GL}_{a}\left(\mathbb{A}_{K}\right)$ whose local component at the place $v$ of $K$ with completion $k$ has Langlands parameter $\tau$. We may assume, thanks to Theorem 1.3, that at some other finite places, say $u_{1}, u_{2}, \cdots, u_{s m}$, the local components $\Sigma_{u_{i}}$ are supercuspidal of level 1 , with parameters $\tau_{u_{i}}$ of the same parity as $\tau$. It is at this stage that we need the restriction that $\tau$ is even dimensional as we are able to construct selfdual representations of $D^{\times} / D^{\times}(1)$ with parameter $\tau \otimes s p_{b}$ only for $\tau$ even dimensional. If the residue characteristic of $k$ is odd, by Proposition 4 of [Pra] any irreducible selfdual representation of the Galois group of $k$ of dimension $>1$ is even dimensional. Hence $\tau$ must be even dimensional unless it is a character of order 2 .

By the work of Moeglin and Waldspurger ([MW]), $\Sigma$ gives rise to a selfdual representation in the residual spectrum of $\mathrm{GL}_{n}\left(\mathbb{A}_{K}\right)$ denoted by $\Sigma[b]$. Next, by Theorem 1.4 concerning the global Jacquet-Langlands correspondence due to Badulescu, the representation $\Sigma[b]$ of $\mathrm{GL}_{n}\left(\mathbb{A}_{K}\right)$ can be transported to an automorphic representation $\Sigma^{\prime}[b]$ of $\mathbb{D}^{\times}\left(\mathbb{A}_{K}\right)$, where $\mathbb{D}$ is the division algebra of index $n$ over $K$ constructed earlier. 
Transporting the automorphic representation $\Sigma[b]$ to $\mathbb{D}^{\times}\left(\mathbb{A}_{K}\right)$, and using the product formula in Theorem 3.1, we get

$$
c(\pi)=[-c(\sigma)]^{s m}=[-c(\sigma)]^{m},
$$

the last equality due to the fact that $s$ is odd because of the condition $(s, 2 r)=1$. This is equivalent to the conclusion of the theorem.

\section{Case III: $\quad b \neq 0$ and $a$ odd:}

If $b$ is also odd, then $D$ has odd index $d$ because $a b=n=m d$, and the proof given above works again, in which we globalize $\sigma$ to an automorphic self-dual representation of $\mathrm{GL}_{a}\left(\mathbb{A}_{K}\right)$, and then use the work of Moeglin and Waldspurger ([MW]), to construct a selfdual representation in the residual spectrum of $\mathrm{GL}_{n}\left(\mathbb{A}_{K}\right)$ denoted by $\Sigma[b]$. As the index of $D$ is odd, we are able to prove that $c(\pi)=c(\pi)^{d}=1$, as before.

It remains to deal with parameters of the form $\sigma=\tau \otimes s p_{b}, n=$ $a b$, with $\tau$ an irreducible, selfdual $a$-dimensional representation of $W_{k}$ with $\mathbf{a}$ odd and $\mathbf{b}$ even. In this case, we need to prove that the representation $\pi^{\prime}$ of $\mathrm{GL}_{m}(D)$ is orthogonal. Note that $m d=n=a b$.

We will begin by proving the assertion assuming $\mathbf{m}=\mathbf{1}$, so that $\pi^{\prime}$ is a (selfdual) representation of $D^{\times}$, with the Brauer invariant of $D$ being $\frac{s}{a b} \in \mathbb{Q} / \mathbb{Z}$ with $(s, a b)=1$.

As $\tau$ is an irreducible selfdual representation of $W_{k}$, it corresponds to a supercuspidal representation $\pi_{\tau}$, say, of $\mathrm{GL}_{a}(k)$. We may globalize $\pi_{\tau}$ (by applying Theorem 1.3) to a selfdual, cuspidal automorphic representation $\Pi$ of $\mathrm{GL}_{a}\left(\mathbb{A}_{K}\right)$ with $\pi_{\tau}$ as its local component at two places $v, w$ of $K$ with completions $K_{v}=k=K_{w}$.

Since $\pi_{\tau}$ is supercuspidal with central character of order $\leq 2$, its parameter can be rendered trivial upon restriction to (the Weil group of) a finite extension of $k$ which is a finite succession of cyclic extensions. Let $E$ be a global extension of $K$, which is a finite succession of cyclic extensions, so that

(i) the place $v$ splits completely in $E$;

(ii) the place $w$ remains inert; and

(iii) the parameter of $\pi_{\tau}$ restricted to $E_{w}$ is the trivial $a$-dimensional representation of $W_{k}$.

Let $\Pi_{E}$ be the base change of $\Pi$ to $E$ (cf. $[\mathrm{AC}]$ ). Let $\mathbb{B}$ be a central division algebra over $E$ of dimension $n^{2}$ over $E$ such that the invariant of $\mathbb{B}$ at $a$ places of $E$ above $v$ is that of $D$, and such that $\mathbb{B}$ is ramified at the places $w$ of $E$ with Brauer invariant $\frac{-s}{b} \in \mathbb{Q} / \mathbb{Z}$, and unramified everywhere else. That there is such a division algebra $\mathbb{B}$ follows from classfield theory. 
By $[\mathrm{MW}], \Pi_{E}$ gives rise to a selfdual representation in the residual spectrum of $\mathrm{GL}_{a b}\left(\mathbb{A}_{E}\right)$ denoted by $\Pi_{E}[b]$. Next, by Theorem 1.4 (of Badulescu), $\Pi_{E}[b]$ of $\mathrm{GL}_{a b}\left(\mathbb{A}_{E}\right)$ can be transported to an automorphic representation $\Pi^{\prime}:=\Pi_{E}^{\prime}[b]$ of $\mathbb{B}^{\times}\left(\mathbb{A}_{K}\right)$, where $\mathbb{B}$ is the division algebra of index $n=a b$ over $E$ just constructed.

By the product formula (Theorem $\mathrm{C}$ ), $c\left(\Pi_{v}^{\prime}\right)^{a}$ equals the sign associated to the representation $\Pi_{w}^{\prime}$ of $\mathrm{GL}_{a}\left(D^{\prime}\right)$ obtained by parabolic induction of the trivial representation of the minimal parabolic of $\mathrm{GL}_{a}\left(D^{\prime}\right)$ (which has parameter $\left(s t_{b}\right)^{a}$ ), where $D^{\prime}$ is a division algebra over $E_{w}$ with Brauer invariant $\frac{-s}{b}$. The representation $\Pi_{w}^{\prime}$ is clearly unitary, and defined over $\mathbb{R}$, hence orthogonal by Lemma 2.1. Thus $c\left(\Pi_{v}^{\prime}\right)=1$.

Now let us consider the case $\mathbf{m}>\mathbf{1}$. Let $M_{m}(D)$ have Brauer invariant $\frac{s}{d}$ with $(s, d)=1$. Replacing $E$, if necessary, by a cyclic extension of degree $s m+1$ in which $v$ splits completely, we may assume that there are places $v=v_{0}, v_{1}, \ldots, v_{s m}$ of $E$ with $E_{v_{j}}=k$ such that for a selfdual, cusp form $\Pi$ on $\mathrm{GL}_{n}\left(\mathbb{A}_{E}\right), \Pi_{v_{j}} \simeq \pi$ for all $j \leq s m$. Now we may, by class field theory, choose a division algebra $\mathbb{D}$ ramified only at these $v_{j}$ such that $\mathbb{D}_{v} \simeq M_{m}(D)$ of Brauer invariant $s / d$, and for every $j \in\{1, \ldots, s m\}, \mathbb{D}_{v_{j}} \simeq B^{\times}$, with $B$ a division algebra over $E_{v_{j}}=k$ of Brauer invariant $-1 / m d$. Applying Theorem 1.4 again, we have a discrete automorphic representation $\Pi^{\prime}:=\Pi_{\mathbb{D}}^{\prime}$ of $\mathbb{D}\left(\mathbb{A}_{E}\right)^{\times}$whose local components have parameter $\sigma$ at each of the $v_{j}$. Since $\mathbb{D}_{v_{j}}$ is a division algebra for each $j \geq 1$, we may apply what we proved above for $m=1$ and conclude that $c\left(\Pi_{v_{j}}^{\prime}\right)=1$ for all $j \in\{1, \ldots, s m\}$. Thus, applying the product formula again, we obtain $c\left(\Pi_{v}^{\prime}\right)=1$, as desired.

To complete the proof in this case, we need to address the issue that the global Jacquet-Langlands correspondence might produce a Speh representation on $\mathrm{GL}_{m}(D)$, whereas the assertion of Theorem A is about a corresponding discrete series representations of $\mathrm{GL}_{m}(D)$. In other words, the local representation $\Pi_{v}^{\prime}$ of $\Pi^{\prime}$ may not be the $\pi^{\prime}$ we started with on $\mathrm{GL}_{m}(D)$ but is related to it by the Aubert-Zelevinsky involution. Nevertheless, we claim that

$$
c\left(\pi^{\prime}\right)=c\left(\Pi_{v}^{\prime}\right) .
$$

To deduce it we appeal to the following proposition, as well as the fact that the Aubert-Zelevinsky involution interchanges Speh-modules with the corresponding generalized Steinberg representations. This involution $\pi_{1} \rightarrow i\left(\pi_{1}\right)$ (cf. [Bdl], section 2.6, for example) is defined on the Grothendieck group of smooth representations of a $p$-adic reductive 
group $G$ as an alternating sum of parabolically induced representations of the various Jacquet modules of $\pi_{1}$, and sends an irreducible to another irreducible $\left|i\left(\pi_{1}\right)\right|$ up to sign.

Proposition 5.2. Let $G$ be a reductive algebraic group over a nonArchimedean local field $k$. Then an irreducible representation $\pi$ is orthogonal if and only if the corresponding irreducible representation $|i(\pi)|$, associated by the Aubert-Zelevinsky involution $i$, is orthogonal.

Proof. The assertion follows by combining Proposition 2.1 with the fact that both induction and the Jacquet functor take real representations to real representations.

We are now finished with the proof of Theorem A, and also Corollary $\mathrm{B}$, which is an immediate consequence.

\section{Rationality}

The question about whether an irreducible selfdual representation is orthogonal or symplectic is part of the more general question about field of definition of a representation. For example, as Lemma 2.1 shows, a selfdual, unitary representation of a $p$-adic group is orthogonal if and only if it is defined over $\mathbb{R}$.

Let $G$ be a group, and $\pi$ an irreducible representation of $G$ over $\mathbb{C}$. Put

$$
\mathcal{G}_{\pi}=\left\{\sigma \in \operatorname{Aut}(\mathbb{C} / \mathbb{Q}) \mid \pi^{\sigma} \cong \pi\right\}
$$

If either $G$ is finite, or $G$ is a reductive $p$-adic group, and $\pi$ is supercuspidal with finite order central character, then $\mathcal{G}_{\pi}$ is known to be a subgroup of finite index of $\operatorname{Aut}(\mathbb{C} / \mathbb{Q})$, and this defines a finite extension $K$ of $\mathbb{Q}$. Call $K$ the field of definition of $\pi$. (If $\pi$ is finite dimensional, it is the field generated by the character values of $\pi$.)

Assume that $\pi$ is finite dimensional. Then associated to $\pi$, there is a division algebra $\mathcal{D}_{\pi}$ with center $K$ (an abelian extension of $\mathbb{Q}$ ) which measures the obstruction to $\pi$ being defined over $K$, called Schur Algebra, thus $\mathcal{D}_{\pi}=K$ if and only if $\pi$ can be defined over $K$.

Now let $\sigma \rightarrow \pi_{\sigma}$ be the local Langlands correspondence between irreducible representations of $\operatorname{Gal}(\bar{k} / k)$ of dimension $n$, and representations of $\mathrm{GL}_{m}(D)$ where $D$ is a division algebra of index $d$ with $d m=n$. Here and in what follows, we normalize the Langlands correspondence by multiplying by the character $x \rightarrow|x|^{(n-1) / 2}$ where $x \in k^{\times}$. This normalized Langlands correspondence, which does not affect rationality (reality!) questions at infinity, is what is Galois equivariant on the coefficients; see for example, Henniart, [Hen2]. 
Question: How are $\mathcal{D}_{\sigma}$ and $\mathcal{D}_{\pi_{\sigma}}$ related? This time the answer a priori might depend not just on the index of the division algebra, but on its class in the Brauer group. However, we propose the following conjecture, suggesting in particular that this is not the case.

Conjecture 6.1. Let $D$ be a division algebra of index $d$ over a p-adic local field $k, \pi$ an irreducible representation of $\mathrm{GL}_{m}(D)$, and $\sigma$ the associated $n$-dimensional representation of the Weil-Deligne group of $k$ for $n=m d$. Let $\mathcal{D}_{\sigma}$ and $\mathcal{D}_{\pi}$ be the associated Schur algebras with center a number field $K$ (which is a cyclotomic field). The algebras $\mathcal{D}_{\sigma}$ and $\mathcal{D}_{\pi}$ are invariant under the action of $\operatorname{Gal}(K / \mathbb{Q})$, and the following holds:

1. If $\pi$ is not selfdual, or if $\pi$ is selfdual with $c(\sigma)=c(\pi)$, then $\mathcal{D}_{\sigma}=\mathcal{D}_{\pi}$.

2. If $\pi$ is selfdual and $c(\sigma)=-c(\pi)$, then the answer depends on the degree $K$ over $\mathbb{Q}$ which is a certain totally real extension of $\mathbb{Q}$. If $[K: \mathbb{Q}]$ is even, then the Brauer invariants of $\mathcal{D}_{\sigma}$ and $\mathcal{D}_{\pi}$ are the same except at the infinite places, where the invariants of $\mathcal{D}_{\sigma}$ and $\mathcal{D}_{\pi}$ differ by $1 / 2$. If $[K: \mathbb{Q}]$ is odd, then in particular there are odd number of places in $K$ over $p$. The invariants of $\mathcal{D}_{\sigma}$ and $\mathcal{D}_{\pi}$ are the same except at the Archimedean places and the places above $p$, where the invariants of $\mathcal{D}_{\sigma}$ and $\mathcal{D}_{\pi}$ differ by $1 / 2$.

One case of the conjecture is especially simple to state. This is when $d$, the index of $D$ is odd, and $\pi$ is a selfdual representation of $\mathrm{GL}_{m}(D)$ for $m$ odd. In this case, irreducible selfdual representations of the Galois group of dimension $n$ exist only in even residue characteristic, cf. [Pra]. We are thus in the tame case $(n, p)=1$, and in this case it can be seen that Galois representations are induced from a character $\theta$ of $L^{\times}$where $L$ is a degree $n$ extension of $k$, with $\theta^{2}=1$, thus $\theta$ takes values in \pm 1 . Therefore in this case the Galois representation is defined over $\mathbb{Q}$. Our theorem B implies that the selfdual representations of $\mathrm{GL}_{m}(D)$ are defined over $\mathbb{R}$, and the discussion in this section refines it to ask the following:

Question 6.2 Let $D$ be a division algebra of odd index over a nonArchimedean local field $k$, and $m>0$ an odd integer. Then, is every selfdual, irreducible representation of $\mathrm{GL}_{m}(D)$ defined over $\mathbb{Q}$ ?

Recently, Bushnell and Henniart have answered this question in the affirmative in $[\mathrm{BH}]$ if either $m=1$ or $d=1$. 


\section{A COnCluding Remark}

One of the reasons the proof of Theorem A is so involved is that we do not know, as of yet, how to simultaneously globalize to a selfdual cusp form on $\mathrm{GL}(n)$ a finite number of selfdual, square-integrable local representations (with parameters of the same parity), when one of the representations is supercuspidal while another is (generalized) Steinberg. The reason is that the method of Poincaré series, here applied to the classical groups to produce a generic cusp form with appropriate local components, does not apply when a local discrete series representation is not integrable, as is the case for the Steinberg representation and its generalizations.

It is known that such a general globalization result will follow from a stabilization of Arthur's twisted trace formula for $\operatorname{GL}(n)$, which is being carried out by Arthur in a forthcoming monograph, "Endoscopic Classification of Representations: Orthogonal and Symplectic groups." Some partial results are found in $[\mathrm{CC}]$.

\section{REFERENCES}

[AC] J. Arthur and L. Clozel. Simple algebras, base change, and the advanced theory of the trace formula, volume 120 of Annals of Mathematics Studies. Princeton University Press, Princeton, NJ, 1989.

[Bdl] A. I. Badulescu. Global Jacquet-Langlands correspondence, multiplicity one and classification of automorphic representations. Invent. Math. $\mathbf{1 7 2}$ (2008), 383-438. With an appendix by Neven Grbac.

[BH] C. Bushnell and G. Henniart. Self-dual representations of some dyadic groups, 2008.

[CC] G. Chenevier and L. Clozel. Corps de nombres peu ramifiés et formes automorphes autoduales, preprint, 2007.

[CKPSS] J. W. Cogdell, H. H. Kim, I. I. Piatetski-Shapiro, and F. Shahidi. Functoriality for the classical groups. Publ. Math. Inst. Hautes Études Sci. (2004), 163-233.

[CPSS] J. W. Cogdell, I. I. Piatetski-Shapiro, and F. Shahidi. Stability of $\gamma$ factors for quasi-split groups. J. Inst. Math. Jussieu 7 (2008), 27-66.

[GJR] D. Ginzburg, D. Jiang, and S. Rallis. On the nonvanishing of the central value of the Rankin-Selberg L-functions. J. Amer. Math. Soc. 17 (2004), 679-722 (electronic).

[HT] M. Harris and R. Taylor. The geometry and cohomology of some simple Shimura varieties, volume 151 of Annals of Mathematics Studies. Princeton University Press, Princeton, NJ, 2001. With an appendix by Vladimir G. Berkovich.

[Hen1] G. Henniart. Une preuve simple des conjectures de Langlands pour GL $(n)$ sur un corps p-adique. Invent. Math. 139 (2000), 439-455.

[Hen2] G. Henniart. Une caractérisation de la correspondance de Langlands locale pour GL(n). Bull. Soc. Math. France 130 (2002), 587-602. 
[JS1] D. Jiang and D. Soudry. The local converse theorem for $\mathrm{SO}(2 n+1)$ and applications. Ann. of Math. (2) 157 (2003), 743-806.

[JS2] D. Jiang and D. Soudry. Generic representations and local Langlands reciprocity law for $p$-adic $\mathrm{SO}_{2 n+1}$. In Contributions to automorphic forms, geometry, and number theory, pages 457-519. Johns Hopkins Univ. Press, Baltimore, MD, 2004.

[MW] C. Mœglin and J.-L. Waldspurger. Le spectre résiduel de GL(n). Ann. Sci. École Norm. Sup. (4) 22 (1989), 605-674.

[Pra] D. Prasad. Some remarks on representations of a division algebra and of the Galois group of a local field. J. Number Theory 74 (1999), 73-97.

[PR] D. Prasad and D. Ramakrishnan. Lifting orthogonal representations to spin groups and local root numbers. Proc. Indian Acad. Sci. Math. Sci. 105 (1995), 259-267.

[SZ] A. J. Silberger and E.-W. Zink. An explicit matching theorem for level zero discrete series of unit groups of $\mathfrak{p}$-adic simple algebras. J. Reine Angew. Math. 585 (2005), 173-235.

[Sou1] D. Soudry. On Langlands functoriality from classical groups to $\mathrm{GL}_{n}$. Astérisque (2005), 335-390. Automorphic forms. I.

[Sou2] D. Soudry. Letter to D. Ramakrishnan, December 2007.

[Vig] M.-F. Vignéras. Correspondances entre representations automorphes de $\mathrm{GL}(2)$ sur une extension quadratique de $\mathrm{GSp}(4)$ sur $\mathbf{Q}$, conjecture locale de Langlands pour GSp(4). In The Selberg trace formula and related topics (Brunswick, Maine, 1984), volume 53 of Contemp. Math., pages 463-527. Amer. Math. Soc., Providence, RI, 1986.

[Zlv] A. V. Zelevinsky. Induced representations of reductive p-adic groups. II. On irreducible representations of GL(n). Ann. Sci. École Norm. Sup. (4) 13 (1980), 165-210.

[Zlb] D. P. Želobenko. Compact Lie groups and their representations. American Mathematical Society, Providence, R.I., 1973. Translated from the Russian by Israel Program for Scientific Translations, Translations of Mathematical Monographs, Vol. 40.

School of Mathematics, Tata Institute of Fundamental Research, Mumbai- 400 005, India.

Email: dprasad@math.tifr.res.in

Department of Mathematics, California Institute of Technology, Pasadena, CA 91125.

Email:dinakar@caltech.edu 